\title{
Performance Analysis of Least Load Multicast Routing for Single Rate Loss Networks
}

\author{
Chi-Chung Cheung \\ Dept. of Computer Science, City University of Hong Kong \\ Danny H.K. Tsang \\ Dept. of Electrical and Electronic Eng., HKUST. \\ Sanjay Gupta \\ GSM Products Division, Motorola \\ Hon-Wai Chu \\ School of Science and Technology, Open University of Hong Kong
}

\begin{abstract}
In this paper, we investigate a state dependent multicast routing algorithm called Least Load Multicast Routing (LLMR), for single rate loss networks. The algorithm is based on Least Load Routing (LLR) concept and the approach is to select the least load links for establishing connections. The networks considered are assumed fully connected. In addition, connection requests are Poisson arrival and the holding times of accepted calls are exponentially distributed. The analytical model that we developed for calculating blocking probabilities is based on the link independence assumption and the Reduced Load Approximation (RLA). Analytical results are compared with simulation results and the agreement is surprisingly good. We find that the effect of link independence assumption is insignificant for the analytical model.
\end{abstract}

\section{Introduction}

Multicasting refers to the ability of a set of more than two nodes or end-users in a communication network to communicate simultaneously with each other. Applications that require multicast capability (either point-to-multipoint (PTM) as in distributional video or multipoint-to-multipoint (MTM) as in video conferencing, online collaboration and others) will be an integral part of future broadband services. Given the popularity of multicast end-user services and applications, we study the problem of multicast call routing in single rate loss networks, in which a connection requires a unit capacity on each link that it is routed. If a route cannot be assigned to a connection request, it is assumed that the request is lost and does not retry. Examples of single rate loss networks include telephone networks and homogeneous VP-based ATM networks.

In this paper, we investigate a state dependent multicast routing algorithm called Least Load Multicast Routing (LLMR), to establish connections by considering the link load of links in single rate loss networks. State dependent ${ }^{1}$ routing of calls (pointto-point) has long been regarded in the telephone industry as a means of increasing call throughput and robustness in the telephone network. We would like to refer the reader to [4] [5] [6] for some of the popular state dependent routing algorithms. A key part of the paper pertains to the development of an analytical model for performance evaluation of the routing algorithm. For this purpose, we also use the well known and often used "link independence assumption" to develop a set of fixed point equations from which a number of performance metrics of interest pertaining to the routing algorithm can be obtained. The analytical results developed are used to engineer routing policies to achieve design objectives (for instance maximize revenue, utilization, and/or meet fairness criteria) and dimension networks. Some initial work in the area of state dependent multicast routing schemes in (single rate) circuit switched networks have been reported in [3] [1] and an analytical model is provided in [2] for symmetrical networks.

The paper is organized as follows. We begin by

\footnotetext{
${ }^{1}$ State refers to a set of variables that describe completely the current status of the network.
} 
presenting in Section 2 the system model and the state dependent multicast routing algorithm. In Section 3 we develop an analytical model to estimate the performance of the routing algorithm. Section 4 presents the numerical results of the model and show its accuracy. Finally, we conclude in Section 5 by summarizing the paper and outlining avenues for future research.

\section{Least Load Multicast Rout- ing Algorithm}

In LLMR, the information used to make a routing decision is based on the states of links, i.e the number of free circuits of links. To establish a connection, the LLMR algorithm tries to (i) use direct links to route the connection request and (ii) maximize network capacity for future connection requests. In order to achieve the above objectives, the LLMR locates a spanning tree consisting of only direct links with the highest possible states. If no spanning tree is available, the connection request is denied.

To illustrate the routing algorithm outlined above, we consider a 4-node network and a connection request with destination set $\{r, a, b, c\}$ with node $r$ as the source node. The steps in the connection establishment procedure are as shown in Figure 1 - the number next to a link denotes its state and links that are included in the connection request are drawn with "thick" lines. Node $c$ is the first one to be added as link $(r, c)$ has 3 free circuits that is larger than that of links $(r, a)$ and $(r, b)$ which is followed by node $a$ (link $(c, a)$ is chosen due to its larger number of free circuits over links $(r, a),(r, b)$ and $(c, b))$. Finally, we choose link $(a, b)$ because the number of free circuits of $(a, b)$ is the largest compared with links $(r, b)$ and $(b, c)$. After iteration 4, the procedure is completed and a tree is established for the connection request.
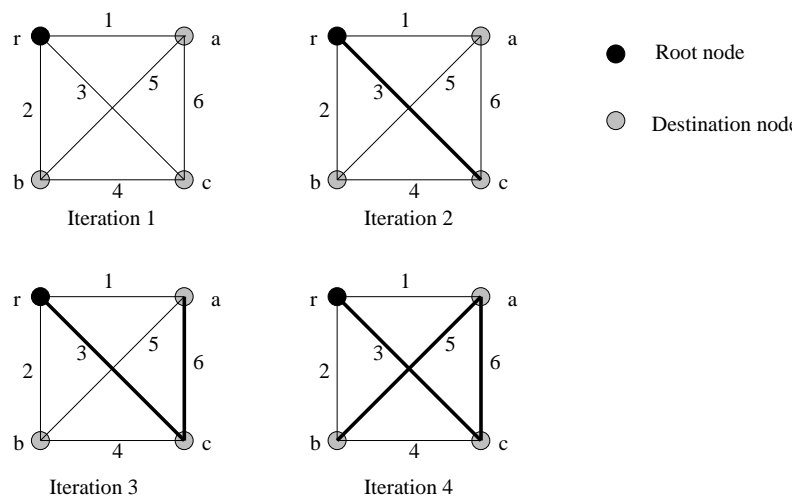

Figure 1: Example to illustrate LLMR

\section{$3 \quad$ Performance Modeling}

Before proceeding further, we would like to introduce necessary definitions and notations. Consider a single rate loss network to be an undirected graph $G=(V, E)$ where $V$ and $E$ are the set of nodes and links respectively. Denote by $C(e), e \in E$, the capacity or number of circuits allocated to link $e$. Consider a connection request $c$ with destination set $S(c)$ (each node $s \in S(c)$ is referred to as a destination of the connection request $c)$. Let $d(c)$ be the number of nodes involved in the connection request $c$, i.e., the size of the destination set. The connection request $c$ requires a connected, acyclic graph (tree) $T(c)=(S(c), E(c))$, where $S(c) \subseteq V(c)$ is chosen and a unit bandwidth is reserved on each link $e \in E(c)$.

Consider a fully connected network with the set of nodes $V$ and links $E$. Connection requests (assumed for the discussion here to have identical bandwidth requirements of 1 bandwidth unit) with destination set, $S(c)$, arrive according to a Poisson process with rate $\lambda_{c}$. It is assumed that the holding times of connections are independent and identically distributed random variables with unit mean.

Throughout we denote by $\pi_{i}^{(e)}, i=0,1,2, \ldots, C(e)$, and $e \in E$, the probability of the link occupancy of the link $e$ being $i$ in steady state. Note that, in LLMR algorithm, the link state represents the number of free circuits. In the analytical model, we denote instead the link state by the number of occupied circuits. We choose the link occupancy to represent the link state for convenience to develop the analytical model. Further, we denote the state dependent arrival rate of the link $e$ in state $i$ by $\Lambda_{i}^{(e)}, i=0, \ldots, C(e)-1$ and $e \in E$. We also denote $\boldsymbol{\Pi}=\left\{\pi^{(e)}: e \in E\right\}$ and $\Lambda=\left\{\Lambda^{(e)}: e \in E\right\}$, where $\pi^{(e)}=\left(\pi_{0}^{(e)}, \ldots, \pi_{C(e)}^{(e)}\right)$ and $\Lambda^{(e)}=\left(\Lambda_{0}^{(e)}, \ldots, \Lambda_{C(e)-1}^{(e)}\right)$.

The analytical models that we develop for calculating blocking probabilities are based on the link independence assumption, i.e. the random variables describing the state of each link are assumed to be independent. This assumption has been used with great success to analyze routing schemes for pointto-point connections (state dependent or otherwise) in a variety of networks; the studies [?] [5] [?] [?] are just a few examples.

The analytical model that we propose is based on the Reduced Load Approximation (RLA), which is an iterative procedure that alternates between the following two steps:

1. Evaluation of link occupancy distributions given the state dependent arrival rates and 
2. Evaluation of state dependent arrival rates given the link occupancy distributions.

Once the consistent set of link occupancy distributions and state dependent arrival rates are obtained, the call blocking probability of each class (or any other performance measure for that matter) can be calculated with little effort.

Part 1: Evaluation of link occupancy distribution given the state dependent arrival rates

Given the state dependent arrival rates, $\Lambda_{i}^{(e)}$, $i=0, \ldots, C(e)-1$, the steady-state link occupancy distribution can be determined by the solution of a one-dimensional birth-death Markov process. Denote by $\gamma^{(e)}(j, l)$ the transition rate of the birthdeath process from state with link occupancy $j$ to state with link occupancy $l$, where $j$ and $l$ are integers, $0 \leq j \leq C(e)-1$ and $0 \leq l \leq C(e)-1$. Thus we have

$$
\gamma^{(e)}(j, j+1)=\Lambda_{j}^{(e)}, \quad 0 \leq j<C(e)-1 ;
$$

and recalling that the mean holding time of each connection is unity, it follows that

$$
\gamma^{(e)}(j, j-1)=j, \quad 0<j \leq C(e)-1 .
$$

Note that $\gamma^{(e)}(j, l)=0$ if $|j-l|>1$. Thus,

$$
\pi_{j}^{(e)}=f_{j}(\boldsymbol{\Lambda}), \quad j=0, \ldots, C(e)-1,
$$

where $\pi_{j}^{(e)}, j=0,1,2, \ldots, C(e)-1$ and $e \in$ $E$, are the steady-state probabilities of the onedimensional birth-death process with rates given by equations (1) and (2).

\section{Part 2: Evaluation of state dependent arrival rates given the link occupancy distributions}

We now discuss the process of evaluating the state dependent arrival rates, $\Lambda$, given the steady state link occupancy distribution $\Pi$. Denote by $\mathcal{Z}(c)$ the set of direct links to be considered in a connection request $c$ with destination set $d(c)$ in a fully connected network and $Z(c)=|\mathcal{Z}(c)|$ (i.e. $Z(c)=d(c)(d(c)-1) / 2)$.

For $e_{k} \in \mathcal{Z}(c), 1 \leq k \leq Z(c)$, let $i_{k}$ be its link state such that it is an integer and $0 \leq i_{k} \leq C\left(e_{k}\right)$, then the probability that the link $e_{k}$ in state $i$ which is selected and included in a connected tree for the call connection request $c$ is

$$
\theta_{i}^{\left(e_{k}\right)}(c)=\sum_{\forall I_{c} \in A_{c}(k, i)} P_{s}\left(e_{k}, C\left(e_{k}\right)-i, I_{c}\right) \prod_{\substack{j=1 \\ j \neq k}}^{Z(c)} \pi_{i_{j}}^{\left(e_{j}\right)}
$$

where $I_{c}=\left(C\left(e_{1}\right)-i_{1}, C\left(e_{2}\right)-i_{2}, \ldots, C\left(e_{Z(c))}\right.\right.$ $\left.i_{Z(c)}\right), A_{c}(k, i)=\left\{I_{c}: i_{k}=i\right.$ and $\left.T(c) \neq \phi\right\}$, and $P_{s}\left(e_{k}, C\left(e_{k}\right)-i, I_{c}\right)$ is the probability that the link $e_{k}$ in state $i$ with $C\left(e_{k}\right)-i$ free circuits is selected when a connection request $c$ arrives and the current state of the links involved is $I_{c}$.

To illustrate the meaning of $P_{s}\left(e_{k}, C\left(e_{k}\right)-i, I_{c}\right)$, consider an example of the 3 -node network shown in Figure 2. Denote by $R(e)$ the residual capacity of link $e$. There are three cases such that link $A$ is possible to be included in a connected tree:

- If $R(A)$ is not the smallest one among three links, $R(A) \neq R(B) \neq R(C)$. and there is at least one free circuits in links $B$ and $C$, then link $A$ must be included;

- If $R(A)$ is the smallest one among them and $R(A)=R(B)$ or $R(A)=R(C)$, then the probability that link $A$ is included in a connected tree is 0.5 because link $A$ is not selected or a second one to be selected;

- If $R(A)=R(B)=R(C)$, then each link has the same probability to be selected and there are two links to be selected in a connected tree. Hence the probability of link $A$ to be included in a connected tree is $2 / 3$.

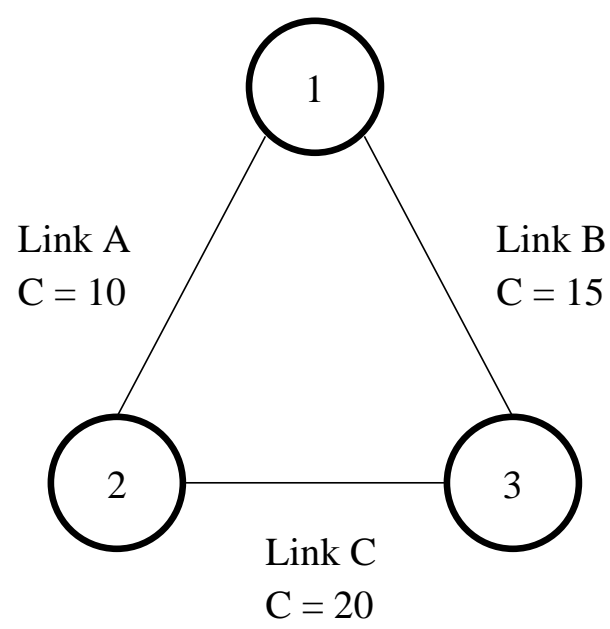

Figure 2: A 3-node network

Thus, we have $P_{s}\left(A, R(A), I_{(1,2,3)}\right)=$

$\begin{cases}1 & \text { if } \quad R(A)>R(B), R(A)>R(C) \\ & \text { and }(R(B) \text { or } R(C) \neq 0) \\ 0.5 & \text { if }(R(A)=R(B) \& R(C)>R(A)) \\ & \text { or }(R(A)=R(C) \& R(B)>R(A)) \\ \frac{2}{3} & \text { if } \quad R(A)=R(B)=R(C) \\ 0 & \text { otherwise }\end{cases}$


Further, by considering all call connection requests that can potentially be carried on link $e$, the total traffic offered to link $e$ in state $i$ is

$$
\Lambda_{i}^{(e)}=\sum_{c \in D_{e}} \lambda_{c} \theta_{i}^{(e)}(c)
$$

where $D_{e}=\{c: e \in \mathcal{Z}(c)\}$ and $\lambda_{c}$ is the arrival rate of the call connection request c. Observe that equations (4) through (6) outline a procedure to obtain $\Lambda_{i}^{(e)}$ from $\Pi$; hence

$$
\Lambda_{i}^{(e)}=g_{i}(\boldsymbol{\Pi}), \quad i=0, \ldots, C(e)-1 \text { and } e \in E,
$$

where $g_{i}(\cdot)$ is a function that is given by equations (4) through (6). The system of equations in (1) and (7) form the Reduced Load Approximations for $\Pi$ and $\Lambda$ :

$$
\begin{gathered}
\Pi=\mathbf{f}(\boldsymbol{\Lambda}) \\
\boldsymbol{\Lambda}=\mathbf{g}(\boldsymbol{\Pi})
\end{gathered}
$$

where $\mathbf{f}$ and $\mathbf{g}$ are the sets of functions $f_{j}$ and $g_{i}$, respectively.

The call blocking probability of call connection request $c$ is then given by

$$
B(c)=\sum_{I_{c} \in \bar{A}_{c}} \prod_{j=1}^{Z(c)} \pi_{i_{j}}^{\left(e_{j}\right)}
$$

where $\bar{A}_{c}=\left\{I_{c}: T(c)=\phi\right\}$, and the call blocking probability of a class with destination set of size $d$ is

$$
B(d)=\frac{\sum_{d(c)=d, \forall c} \lambda_{c} B(c)}{\sum_{d(c)=d, \forall c} \lambda_{c}} .
$$

Hence the normalized revenue loss can be expressed as,

$$
E(\mathrm{loss})=\frac{\sum_{d=2}^{D}(d-1) \lambda_{d} B(d)}{\sum_{d=2}^{D}(d-1) \lambda_{d}} .
$$

The computational requirements of the above approximation procedure are reasonable. Take our numerical results to be presented in Section 4 as an example. Each analytical result obtained requires only one or two minutes on a Sun Spare 5 machine, while a simulation run normally requires overnight on the same machine. The following algorithm outlines the procedure to obtain the values of $\Pi$ and $\Lambda$ :

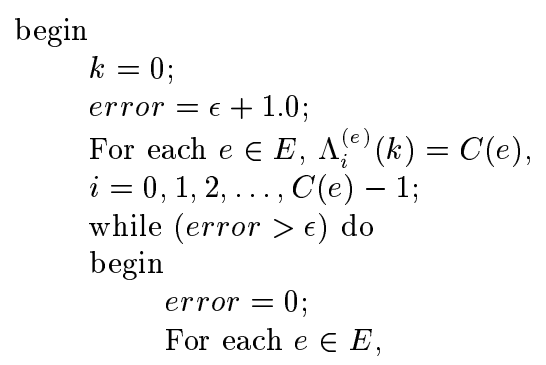

$$
\begin{aligned}
& \text { begin } \\
& \qquad \begin{aligned}
\boldsymbol{\Pi}^{(e)}(k+1) & =\mathbf{f}\left(\boldsymbol{\Lambda}^{(e)}(k)\right) ; \\
\boldsymbol{\Lambda}^{(e)}(k+1) & \mathbf{g}\left(\boldsymbol{\Pi}^{(e)}(k+1)\right) ; \\
\text { error }=\max _{i=0}^{C^{(e)}}\left(\mid \pi_{i}^{(e)}(k+1)\right. & \left.-\pi_{i}^{(e)}(k) \mid, \text { error }\right) ;
\end{aligned}
\end{aligned}
$$

end;

end;

$$
k \leftarrow k+1
$$

end,

where $\pi_{i}^{(e)}(n), \Lambda_{i}^{(e)}(n), \boldsymbol{\Pi}^{(e)}(n)$ and $\boldsymbol{\Lambda}^{(e)}(n)$ are the values of $\pi_{i}^{(e)}, \Lambda_{i}^{(e)}, \boldsymbol{\Pi}^{(e)}$ and $\Lambda^{(e)}$ after $n$ iterations, respectively. $\epsilon$ is the upper bound of an acceptable error, which is set as $10^{-4}$ in the next section.

\section{Numerical Results}

In this section, we present numerical and simulation results to illustrate the performance of our proposed algorithm and verify the accuracy of the analytical models developed. We consider a fully connected network with 20 nodes. The capacity of each link is randomly generated between 10 and 20 bandwidth units or circuits.. Three kinds of connection requests are considered and their destination sizes are 2, 3 and 4 (i.e. $D=4$ ).

We assume the loading of unicast connection is equal to the total loading of multicast connections, i.e. in our case, $L_{2}=L_{3}+L_{4}$ where $L_{d}$ is defined as the loading of class $d\left(L_{d}=(d-1) \lambda_{d}\right)$. For convenience we define the normalized network load, $L$, as the ratio of total offered load to total network capacity, i.e.,

$$
L=\frac{\sum_{d=2}^{D} L_{d}}{\sum_{e \in E} C(e)} .
$$

For each simulation run, the simulation run was terminated after $10^{6}$ connection requests had been generated and the initial $10 \%$ of each run was discarded to avoid the transient effect. The vertical lines about each point indicate the $95 \%$ percent confidence interval. For each analytical result, the iterative procedure will be terminated if the difference between the current and previous values of all $\pi_{i} \mathrm{~s}$ are less than $10^{-4}$, i.e. $\left|\pi_{i}^{(e)}(k+1)-\pi_{i}^{(e)}(k)\right|<10^{-4}$ for each $e \in E$ where $\pi_{i}^{(e)}(k)$ is the value of $\pi_{i}^{(e)}$ after $k$ iterations. In our numerical examples, the iterative procedure always converges to the solution in less than 20 iterations.

Figure 3 shows the accuracy of the analytical models of LLMR. The agreement of simulation and analytical results is observed to be surprisingly good. The high accuracy of the call blocking probability of class 2 shows that the calculations of link 
occupancy distributions and state dependent arrival rates are not affected by the link independence assumption. We also find that the accuracy of the call blocking probability worsens if its destination size becomes larger. It is because, from equation (9), we apply the link independence assumption in the calculation of the call blocking probability. However, the links that involved in a multicast call connection is depended. When the destination size increases, the number of links involved in a call connection increases and hence the link dependence becomes more significant.

Figure 4 shows that when the ratio of the loading of unicast connection to that of multicast connections increases, the error of call blocking probability of class 4 drops significantly and hence it indicates that the error of the calculation of the call blocking probability is due to the link independence assump. tion.

\section{Conclusions}

In this paper, we have investigated the state dependent multicast routing algorithm for single rate loss networks. An analytical model for the routing algorithm has been developed. We have compared the simulation and analytical results, and found that the agreement is surprisingly good. We also founc that the effect of link independence assumption is insignificant for the calculation of state dependent arrival rates and link occupancy distributions.

Substantial work is already in progress to develop an analytical model of the algorithm with alternative nodes. We are also trying to migrate the algorithm to ATM networks and to develop the corresponding analytical model.

\section{References}

[1] C.C. Cheung, H.W. Chu, D.H.K. Tsang, anc S. Gupta. Least loaded first multicast routinধ in single rate loss networks. In Proceedings $o$. the IEEE International Conference of Commu nications, pages 513-517, 1996.

[2] C.C. Cheung, D.H.K. Tsang, and S. Gupta State dependent multicast routing for single rate loss networks. In Proceedings of the Inter. national Conference on Telecommunications, pages 1425-1430, 1997.

[3] R. H. Hwang. Adaptive multicast routing in single rate loss networks. In Proceedings of the IEEE INFOCOM, pages 571-578, 1995.
[4] P. B. Key and G. A. Cope. Distributed dynamic routing schemes. IEEE Communications Magazine, vol. 28:54-64, October 1990.

[5] D. Mitra, R. J. Gibbens, and B. D. Huang. State-dependent routing on symmetric loss networks with trunk reservations, I. IEEE Transactions on Communications, pages 400-411, February, 1993.

[6] T. J. Ott and K. R. Krishnan. State-dependent routing of telephone schemes and the use of separable routing schemes. In Proceedings of the International Teletraffic Congress, 1985.

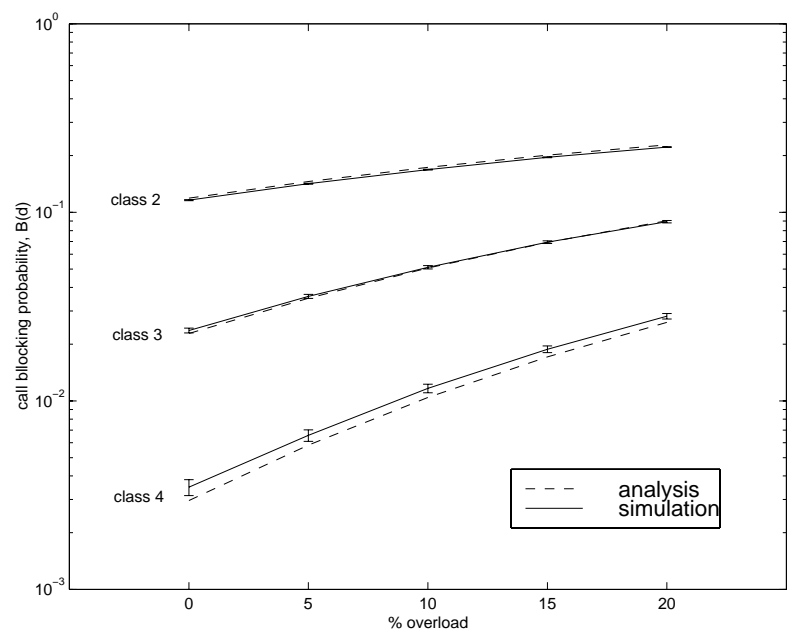

Figure 3: Call blocking probability versus network loading

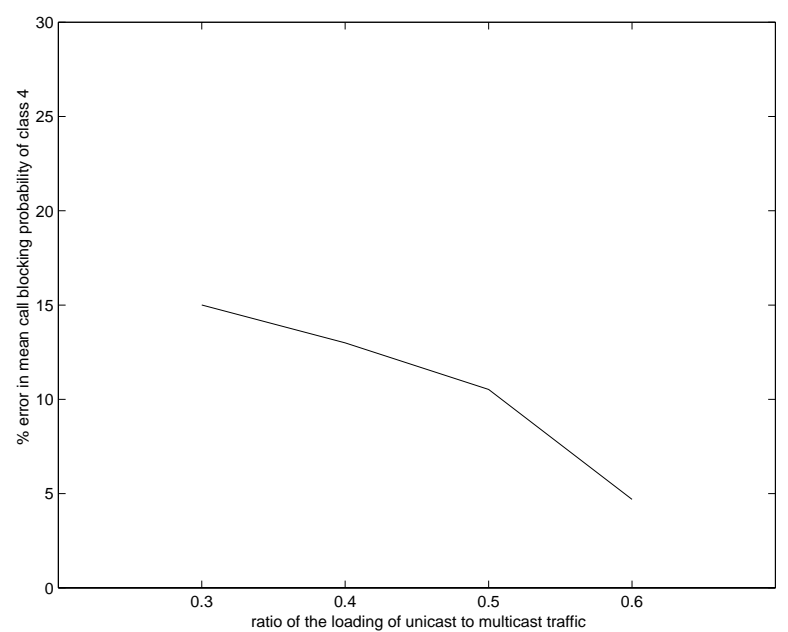

Figure 4: \% error in mean call blocking probability of class 4 versus the ratio of the loading of unicast connections to that of multicast connections 